\title{
Zu diesem Sonderheft
}

\section{ZfE}

\author{
Rainer Bromme • Manfred Prenzel
}

(C) Springer Fachmedien Wiesbaden 2014

Ausgangspunkt dieses Sonderheftes der Zeitschrift für Erziehungswissenschaft ist ein Gutachten, das die Herausgeber (gemeinsam mit M. Jaeger) für das BMBF erstellt haben und das - etwas überarbeitet- den Auftakt dieses Bandes bildet. Es geht darin um den Zusammenhang zwischen evidenzbasierter Bildungspolitik sowie Bildungspraxis und der Kommunikation zwischen Wissenschaft und Öffentlichkeit.

Mit drei Fallbeispielen werden in diesem ersten Beitrag exemplarisch die Prozesse der Generierung von Evidenz und der Rezeption von Forschungsergebnissen dargestellt. Am Beispiel der gewalthaltigen Computerspiele wird gezeigt, dass empirische Forschung empirische Evidenz (bis zu kausal relevanten Einflussfaktoren) liefern kann, die gleichzeitig belastbar und dennoch unabgeschlossen ist. Die öffentliche Debatte dazu wird nachgezeichnet. Die Forschung zu diesem Thema illustriert, dass Vorläufigkeit wissenschaftlicher Evidenz der Normalfall wissenschaftlicher Forschung ist, der jedoch einer Nutzung der Erkenntnisse in Bildungspolitik und Bildungsadministration nicht entgegensteht. Die Forschung kann also zur Versachlichung der jeweiligen Debatten beitragen. Allerdings setzt dies methodisch angemessene Forschungssynthesen und ein sozialwissenschaftlich fundiertes Verständnis der öffentlichen Debatten und Auffassungen zu dieser Thematik voraus.

Prof. Dr. R. Bromme $(\bowtie)$

Institut für Psychologie, Universität Münster,

Fliednerstr. 21,

48149 Münster, Deutschland

E-Mail: bromme@uni-muenster.de

Prof. Dr. M. Prenzel

Susanne Klatten-Stiftungslehrstuhl für Empirische Bildungsforschung,

TU München School of Education,

Schellingstr. 33,

80799 München, Deutschland

E-Mail: Manfred.prenzel@tum.de 
Auch die beiden anderen Beispiele der Wirkungen von Klassengröße und die Frage nach den leistungsbezogenen Auswirkungen von unterschiedlichen Schulformen zeigen, dass die Evidenz nicht unmittelbar aus den Forschungsergebnissen ausgelesen werden kann, sondern methodisch elaborierte Forschungssynthesen erfordert. Und sie zeigen, dass empirische Evidenz zwar heuristische Anregungen zur Gestaltung bestimmter bildungspolitischer Maßnahmen liefert, dass diese aber letztlich auch eine politisch- normative Begründung benötigen. Die drei Beispiele haben aber auch gemeinsam, dass viele Annahmen, die in die dann notwendige politische Diskussion eingehen, durchaus empirisch und konzeptuell mit den Methoden der Bildungsforschung überprüft werden können.

Im Ergebnis empfehlt dieser Beitrag (bzw. das Gutachten) die Ausarbeitung eines Forschungsprogramms mit zwei Themenschwerpunkten: im Bereich der empirischen Bildungsforschung: 1) Forschung und Entwicklung zur Erstellung von evaluativen Forschungssynthesen. 2) Forschung und Entwicklung zur Kommunikation zwischen Wissenschaft und Öffentlichkeit zu Themen der empirischen Bildungsforschung.

Die weiteren Beiträge in diesem Sonderheft stellen exemplarisch mögliche Fragestellungen und Ansätze zu diesen beiden Themenbereichen vor. Diese Aufsätze vertiefen und ergänzen die oben skizzierten Argumente.

Evidenzbasiertes Bildungspraxis setzt die Verfügbarkeit solcher Evidenz voraus, deshalb sind zwei Beiträge den theoretischen und methodischen Fragen der Ergebnisakkumulation gewidmet. Andreas Beelmann diskutiert methodische Fragen der Ergebnisakkumulation durch Metaanalysen. Hans Anand Pant illustriert seine Darstellung der Möglichkeiten und Grenzen metaanalytischer Evidenzakkumulation in der Bildungsforschung durch eine kritische Diskussion der aktuellen und viel diskutierten Metaanalyse von John Hattie zu den Merkmalen erfolgreichen Unterrichts.

Die Darstellung und die Rezeption von Forschungsbefunden werden durch die Erwartungen und durch die bereits vorhandenen Positionen der Vermittler (z. B. Journalisten) wie auch der Rezipienten beeinflusst. Dies zeigen Mario Gollwitzer, Tobias Rothmund, Christoph Klimmt, Peter Nauroth und Jens Bender am Beispiel der Debatte um gewalthaltige Computerspiele. Ihr Beitrag illustriert zugleich wie ein empirischer psychologischer Zugang zum Verhältnis Wissenschaft und Öffentlichkeit aussehen kann. Sabrina Heike Kessler, Lars Guenther und Georg Ruhrmann beleuchten mit epistemologischen Überzeugungen eine der kognitiven Voraussetzungen der Rezeption von Wissenschaft und zugleich weisen sie daraufhin, dass Wissenschaftsvermittlung nicht nur textbasiert (Printmedien, Internet) sondern auch in anderen Medien erfolgt.

Der Beitrag von Tonia Bieber, Kerstin Martens, Dennis Niemann und Michael Windzio diskutiert aus einer soziologischen Perspektive den Zusammenhang von empirischer Bildungsforschung (am Beispiel der PISA Studien) und Bildungspolitik.

Die Beiträge dieses Sonderheftes sind noch keine Landkarten eines solchen Forschungsprogramms, aber es sind Reiseberichte von einzelnen Erkundungen und sie lassen den Zusammenhang beider Forschungsfragen erkennen.

Wir danken vier Kolleginnen und Kollegen für sachkundige Reviews und Frau Stefanie Nordenbrock (Münster) für die technische Unterstützung bei der Aufbereitung der Manuskriptvorlagen. 